\title{
Effect of Bacillus mesonae H20-5 Treatment on Rhizospheric Bacterial Community of Tomato Plants under Salinity Stress
}

\author{
Shin Ae Lee ${ }^{1,2}$, Hyeon Su Kim¹, Mee Kyung Sang', Jaekyeong Song ${ }^{1}$, and Hang-Yeon Weon ${ }^{1 *}$ \\ ${ }^{1}$ Agricultural Microbiology Division, National Institute of Agricultural Sciences, Rural Development Administration, \\ Wanju 55365, Korea \\ ${ }^{2}$ Division of Genetic Diversity, Honam National Institute of Biological Resources, Mokpo 58762, Korea
}

(Received on October 21, 2021; Revised on November 18, 2021; Accepted on November 21, 2021)

Plant growth-promoting bacteria improve plant growth under abiotic stress conditions. However, their effects on microbial succession in the rhizosphere are poorly understood. In this study, the inoculants of Bacillus mesonae strain $\mathbf{H 2 0 - 5}$ were administered to tomato plants grown in soils with different salinity levels (EC of 2,4 , and $6 \mathrm{dS} / \mathrm{m}$ ). The bacterial communities in the bulk and rhizosphere soils were examined 14 days after H20-5 treatment using Illumina MiSeq sequencing of the bacterial 16S rRNA gene. Although the abundance of $\mathrm{H20-5}$ rapidly decreased in the bulk and rhizosphere soils, a shift in the bacterial community was observed following $\mathbf{H 2 0 - 5}$ treatment. The variation in bacterial communities due to $\mathbf{H 2 0 - 5}$ treatment was higher in the rhizosphere than in the bulk soils. Additionally, the bacterial species richness and diversity were greater in the $\mathbf{H 2 0 - 5}$ treated rhizosphere than in the control. The composition and structure of the bacterial communities varied with soil salinity levels, and those in the $\mathrm{H20-5}$ treated rhizosphere soil were clustered. The members of Actinobacteria genera, including Kineosporia, Virgisporangium, Actinoplanes, Gaiella, Blastococcus, and Solirubrobacter, were enriched in the H20-5 treated rhizosphere soils. The microbial co-occurrence net-

*Corresponding author.

Phone) +82-63-238-3042, FAX) +82-63-238-3834

E-mail)why@korea.kr

Handling Editor : Kihyuck Choi

(c) This is an Open Access article distributed under the terms of the Creative Commons Attribution Non-Commercial License (http:// creativecommons.org/licenses/by-nc/4.0) which permits unrestricted noncommercial use, distribution, and reproduction in any medium, provided the original work is properly cited.

Articles can be freely viewed online at www.ppjonline.org. work of the bacterial community in the $\mathrm{H20-5}$ treated rhizosphere soils had more modules and keystone taxa compared to the control. These findings revealed that the strain H20-5 induced systemic tolerance in tomato plants and influenced the diversity, composition, structure, and network of bacterial communities. The bacterial community in the $\mathbf{H 2 0 - 5}$ treated rhizosphere soils also appeared to be relatively stable to soil salinity changes.

Keywords : Bacillus mesonae, bacterial community, rhizosphere, salinity, tomato

Soil salinity is a critical environmental factor that limits plant development and growth. Low precipitation and high evaporation rates resulting from climate change and poor agricultural management have resulted in the expansion of salinized agricultural areas. It is expected that $50 \%$ of agricultural areas will be salinized by 2050 (Shrivastava and Kumar, 2015). Soil salinity levels indicate the electrical conductivity (EC) of the soil; higher levels of soil EC $(>4 \mathrm{dS} / \mathrm{m}$ ) reduce the growth and yield of most crop plants (Jamil et al., 2011; Munns, 2005). Soil EC is also a critical edaphic factor that shapes the bacterial community; Lee et al. observed a significant relationship between bacterial communities in the tomato rhizosphere and soil EC levels (Kim et al., 2016; Lee et al., 2019). Using microorganisms to enhance crop tolerance to salinity stress has been considered an alternative strategy to improve crop production. The physiological effects of plant growth-promoting bacteria (PGPB) on plants under abiotic stresses have been studied. However, there is limited understanding on how PGPB treatment affects the soil microbial composition under abiotic stress conditions. 
Soil harbors a high microbial diversity, whose rhizospheric composition is influenced by plants through their root exudates (Bulgarelli et al., 2013; Compant et al., 2019). A subset of the microorganisms in the rhizosphere also penetrates the plant tissues and colonizes the endosphere depending on the plant's innate immune system (Bulgarelli et al., 2013). Hence, plants have distinct microbial communities depending on their rhizo-compartments; additionally, the composition and structure of the microbial communities affect plant phenotypes such as flowering time, disease suppression, and drought tolerance (PankeBuisse et al., 2015; Wei et al., 2019; Zolla et al., 2013). The manipulation of plant rhizosphere microbiome can improve plant performances (Toju et al., 2018).

PGPB are beneficial microorganisms that colonize the rhizosphere and endosphere. These bacteria increase plant nutrient acquisition, suppress plant pathogens, and alleviate biotic and abiotic stress damage (Backer et al., 2018; Glick, 2012). PGPB modulate environmental stresses in plants by reducing ethylene levels using 1-aminocyclopropane1-carboxylate deaminase and producing indole-3-acetic acid, cytokinin, and volatile organic compounds. PGPB also reduce the reactive oxygen species levels using antioxidants and accumulate osmolytes in plant cells (Glick, 2012; Yang et al., 2009). Yang et al. (2009) proposed the term induced systemic tolerance (IST) for PGPB-induced physical and chemical changes that enhance plant tolerance to abiotic stresses. The genera Azospirillum, Azotobacter, Bacillus, Burkholderia, Pseudomonas, Streptomyces, and Rhizobia are well-known PGPB that have been commercialized for agricultural management (Glick, 2012).

Microbial inoculation can influence the composition and structure of microbial communities in the rhizosphere and endosphere, as observed when Bacillus velezensis NJNUZ9 was inoculated into pepper, enhancing bacterial diversity in the rhizosphere (Trabelsi and Mhamdi, 2013; Zhang et al., 2019b). Treatments with bio-organic fertilizers, which are the mixtures of organic matter and PGPB, alter microbial communities in the rhizosphere, thereby suppressing plant diseases and improving the soil chemical properties including organic carbon, nitrogen, and potassium (Liu et al., 2018; Tao et al., 2020; Wang et al., 2015; Xue et al., 2015; Zhang et al., 2019a). Three different Bacillus species (B. cereus, B. subtilis, and B. amyloliquefaciens) modulate bacterial diversity, evenness, and composition of the bacterial community in the root endosphere (Gadhave et al., 2018).

Previous studies have revealed that Bacillus mesonae strain H20-5, isolated from soil-cultivated lettuce, enhanced tolerance to salinity stress in tomato plants (Sawant et al.,
2019; Yoo et al., 2019b). The plants treated with H20-5 have exhibited significantly decreased electrolyte leakage and accumulated proline, abscisic acid (ABA), and antioxidant enzyme activities compared to untreated plants (Yoo et al., 2019b). Strain H20-5 has also upregulated 9-cisepoxycarotenoid dioxygenase 1 (NCEDI) and abscisic acidresponse element-binding protein 1 (AREB1), which are involved in ABA biosynthesis and signaling, respectively (Yoo et al., 2019b). Field tests revealed that strain H20-5 improves fruit productivity and quality of tomato, strawberry, and cucumber (Yoo et al., 2019a). Whole genome analysis revealed that $\mathrm{H} 20-5$ possesses putative functional genes involved in biofilm formation and in the biosynthesis of auxin, proline, and spermidine (Sawant et al., 2019).

This study identified the effects of administering Bacillus mesonae strain H20-5 on bacterial communities in the bulk and rhizosphere soils of tomato (Solanum lycopersicum L.) plants grown under different soil salinity levels. To analyze bacterial communities, we conducted Illumina MiSeq sequencing of 16S rRNA genes and examined bacterial species richness and diversity, bacterial community structure, enriched taxa, and microbial network topology using bioinformatics tools.

\section{Materials and Methods}

Plant growth conditions and treatment of bacterial inoculant. The soil used in this study was collected from an experimental vegetable field at the National Institute of Agricultural Sciences (35 $\left.49^{\prime} 33.08^{\prime \prime N}, 127^{\circ} 2^{\prime} 38.82^{\prime \prime E}\right)$. The soil was air dried in the shade for a week and passed through a 2-mm sieve. The soil EC value was $0.4 \mathrm{dS} / \mathrm{m}$. To establish soils with different salinity levels, we adjusted the soil $\mathrm{EC}$ values to 2, 4, and $6 \mathrm{dS} / \mathrm{m}$ by supplementing diluted $-1,000 \mathrm{kPa}$ salinity solutions (Polonenko et al., 1986) at ratios of 2.4:7.6, 5.5:4.5, and 8.6:1.4 (-1,000 $\mathrm{kPa}$ salinity solution:water), respectively. Tomato (Solanum lycopersicum "Juiken") seeds were germinated on wet filter papers for 3 days and sown in plastic pots $(5 \mathrm{~cm}$ in diameter) filled with $50 \mathrm{~g}$ of dried soil. Diluted saline solutions $(10 \mathrm{ml})$ were added. To maintain the initial soil moisture levels, the pots were watered daily according to their respective weights.

Bacillus mesonae strain H20-5 (Yoo et al., 2019b) was cultured in R2A broth at $28^{\circ} \mathrm{C}$ and $150 \mathrm{rpm}$ for 2-3 days. After centrifugation at $8,000 \mathrm{rpm}$ for $10 \mathrm{~min}$, the supernatants were removed, and the cells were resuspended using $10 \mathrm{mM} \mathrm{MgSO} \mathrm{M}_{4}$ such that $\mathrm{OD}_{600}=0.25\left(>10^{8} \mathrm{cfu} / \mathrm{ml}\right)$. A week after sowing the tomato seeds in saline soils, $5 \mathrm{ml}$ of the $B$. mesonae $\mathrm{H} 20-5$ suspension was added. $\mathrm{MgSO}_{4}(10$ 
$\mathrm{mM}$ ) was used as the untreated control.

Soil $\mathrm{pH}$ and $\mathrm{EC}$ values were measured using a $\mathrm{pH}$ meter (CyberScan pH1500, EUTECH, Singapore) and an EC meter (D-54, Horiba, Kyoto, Japan), respectively, after shaking the soil:water $(1: 5 \mathrm{w} / \mathrm{v})$ mixture for $30 \mathrm{~min}$ at 200 rpm.

Fresh weight measurement and rhizosphere sample preparation. After two weeks of bacterial inoculum treatment, we measured the fresh weight of tomato seedlings and collected bulk and rhizosphere soil samples. Loose soil without tomato roots was carefully collected as the bulk soil sample. Subsequently, the tomato roots were vigorously shaken by hand to remove adherent soil particles. The roots with firmly attached soil were placed into 50-ml tubes containing $0.85 \% \mathrm{NaCl}$ and shaken vigorously using a shaker (CUTE MIXER CM-1000, EYELA, Tokyo, Japan) for $30 \mathrm{~min}$. The roots, which contained soil and saline water, were removed from the tubes and were centrifuged at $8,000 \mathrm{rpm}$ for $15 \mathrm{~min}$. The supernatants were removed, and the remaining soil samples were used as the rhizosphere samples.

DNA extraction and quantitative PCR analysis. Bulk and rhizosphere soil samples ( $0.5 \mathrm{~g}$ each) were used in triplicates for DNA extraction. The Power Soil DNA Isolation Kit (MoBio, Carlsbad, CA, USA) was used for extraction according to the manufacturer's instructions, and the samples were subsequently pooled. Prior to DNA extraction, soil samples derived from individual tomato plants were prepared separately and pooled together to minimize variation. The extracted DNA was quantified using the Qubit dsDNA BR Assay Kit (Invitrogen, Carlsbad, CA, USA). The abundance of total bacteria and strain H20-5 was quantified using real-time PCR (Bio-Rad CFX96 System, Hercules, CA, USA) using the primer pair $16 \mathrm{~S} 338 \mathrm{~F}$ (5'-ACTCCTACGGGAGGCAG-3')/518R (5'-WTTACCGCGGCTGCTGG-3') and InsK1F (5'-ACTTGCCCACGGTA TGAACG-3')/InsK1R (5'-GATCTAGTAAAGGTCGCTGGTATTG-3'), respectively.

Bacterial 16S rRNA gene amplicon sequencing. To generate bacterial libraries, universal 16S rRNA gene primers (799F: 5'-AACMGGATTAGATACCCKG-3' and 1193R: 5'-ACGTCATCCCCACCTTCC-3') were used for PCR amplification. These target-specific primers were attached to the Nextera consensus and adaptor sequences with the forward (5'-TCGRCGGCAGCGTC-AGATGTGTATAAGAGACAG-target sequence-3') and reverse (5'-GTCTCGTGGGCTCGG-AGATGTGTATAAGAGACAG-target sequence-3') primers for the first round of PCR amplification. The PCR conditions were as follows: initial denaturation at $94^{\circ} \mathrm{C}$ for $3 \mathrm{~min}, 25$ cycles of denaturation at $94^{\circ} \mathrm{C}$ for $30 \mathrm{~s}$, annealing at $55^{\circ} \mathrm{C}$ for $30 \mathrm{~s}$, extension at $72^{\circ} \mathrm{C}$ for $30 \mathrm{~s}$, and final extension at $72^{\circ} \mathrm{C}$ for $5 \mathrm{~min}$. Following initial amplification of the bacterial target sequences, the library size was verified via agarose gel electrophoresis, and the PCR products were cleaned using Agencourt AMPure XP (Beckman Coulter, Inc., Miami, FL, USA). A second round of PCR amplification was conducted with primers containing Illumina dual indices and sequencing adapters, namely, S502F (5'-AATGATACGGCGACCACCGAGATCTACAC-55555555-TCGTCGGCAGCGTC-3') and N701R (5'-CAAGCAGAAGACGGCATACGAGAT77777777-AGTCTCGTGGGCTCGG-3'), under the same conditions as those used for the first round of PCR. The PCR products were cleaned using Agencourt AMPure XP (Beckman Coulter, Inc.) and quantified using the Quant-iT PicoGreen dsDNA Assay Kit (Invitrogen). Purified amplicon libraries were pooled at equimolar concentrations and sequenced with an Illumina MiSeq system using the MiSeq Reagent Kit v3 (Illumina Inc., San Diego, CA, USA). Bacterial DNA samples were sequenced at the National Instrumentation Center for Environmental Management (NICEM; Republic of Korea). Sequence data are available in the GenBank SRA database under the BioProject accession number PRJNA674044.

Sequence data processing. The sequences obtained from the MiSeq platform were processed using the UPARSE pipeline (ver. 9.1.13 i86linux64, http://www.drive5.com/ usearch) (Edgar, 2013). The paired-end reads were merged when the number and ratio of mismatches in the overlap region were $<10 \mathrm{bp}$ and $10 \%$, respectively. Low-quality reads that were above the expected error threshold $(>1)$ and short reads $(<300 \mathrm{bp})$ were removed. To minimize the impact of sequencing artifacts, singletons were removed from the datasets. Chimeric sequences were removed using the UCHIME de novo algorithm. The remaining high-quality sequences were clustered into operational taxonomic units (OTUs) with 97\% identity using the UPARSE algorithm. Representative sequences of bacterial and archaeal OTUs were classified using the naïve Bayesian classifier (Wang et al., 2007) based on the Ribosomal Database Project (RDP) database (Cole et al., 2014) with a $60 \%$ confidence threshold. OTUs affiliated with chloroplasts and nonbacterial cells were subsequently removed from the bacterial OTU table. To assess alpha-diversity indices, sequence reads of bulk soil and rhizosphere samples were rarefied to 14,000 reads, and six indices including coverage, number 
of OTUs, Chao1, ACE, Shannon, and Inverse Simpson were subsequently calculated using Mothur (version 1.29.1, http://www.mothur.org) (Schloss et al., 2009).

Data analyses. Statistical analyses in this study were performed using R 3.3.1 (R Development Core Team, 2014). The OTU abundances in the dataset were normalized using Hellinger transformation (Legendre and Gallagher, 2001) using the "decostand" function of the vegan package (Oksanen et al., 2013) in R. Subsequently, non-metric multidimensional scaling (NMDS) analysis was performed based on the Bray-Curtis dissimilarity matrix using the "metaMDS" function of the vegan package. The differences in community structure between rhizo-compartments, soil EC levels, and H20-5 treatment were tested by ANOSIM (Clarke, 1993) using the "anosim" function and permutational multivariate analysis of variance (PERMANOVA) (Anderson, 2001) using the "adonis" function. These calculations were based on the Bray-Curtis dissimilarity matrix, which was calculated using the "vegdist" function within the vegan package. To identify the OTUs that were specifically abundant due to $\mathrm{H} 20-5$ treatment, indicator species analysis was conducted using the "multipatt" function with the "r.g" option in the indispecies package (De Cáceres and Legendre, 2009), and OTUs with high indicator values $(>0.5)$ and high abundances $(>0.1 \%$ relative abundance) were screened from the results.

Network analysis was conducted using the Molecular Ecological Network Analyses pipeline based on random matrix theory (RMT) at the University of Oklahoma's Institute for Environmental Genomics web server (http://ieg2. ou.edu/MENA/). The details of the process are provided by Shi et al. (2016). The input datasets were separated using H20-5 treatment and non-treatment. For network construction, the following options were used: OTUs detected in all nine samples were used, 0.01 was filled in the blanks with paired valid values, logarithm values were obtained, Pearson correlation coefficient was used for correlation analysis, and calculation was made by decreasing the cutoff from the top using only Poisson regression. A similarity threshold was selected automatically using the RMT-based approach to define the adjacency matrix. The topological descriptors were measured using R package $Q u A C N$ (Mueller et al., 2011). The modularity of the network was calculated using the greedy modularity optimization method. Subsequently, the within-module connectivity ( $\mathrm{Zi})$ and among-module connectivity (Pi) of each node were examined, and topological roles were classified based on the values of $\mathrm{Zi}(2.5)$ and $\mathrm{Pi}(0.62)$.

\section{Results}

Strain H20-5 promotes tomato growth under salinity stress conditions. To establish soils with different salinity levels, we sowed germinated tomato seeds in air-dried soils and drenched the soils in saline solutions of different concentrations. We confirmed that the soils maintained the EC levels at 2, 4, and $6 \mathrm{ds} / \mathrm{m}$, respectively, and soil $\mathrm{pH}$ were between 6.0-6.5 (Supplementary Fig. 1). We treated the inoculant of strain H20-5 7 days after sowing germinated tomato seeds. The fresh weight of the tomato plants was measured 14 days after H20-5 treatment. Consistent with previous results (Yoo et al., 2019a), we confirmed that tomato growth was higher in the H20-5 treatment group than in the controls at different soil salinity levels (Supplementary Fig. 2).

To examine the sustained abundance of strain $\mathrm{H} 20-5$ in soils after H20-5 treatment, the bulk and rhizosphere soils were separately collected at $0,2,4,7$, and 14 days after treatment, and the copy numbers of strain H20-5 were measured via real-time quantitative reverse transcription PCR with H20-5 specific primers. On the day of the H20-5 treatment, the copy number of strain H20-5 was high in the bulk soil when compared to the control, at EC values of 2, 4 , and 6 ds/m (Supplementary Fig. 3). However, the abundance of H20-5 rapidly decreased and became insignificant at 14 days after treatment. The abundance of total bacteria quantified by the 16S rRNA gene was not significantly different between the H20-5 treatment and control (Supplementary Fig. 3).

Effect of strain H20-5 on bacterial richness and diversity. To investigate the effects on bacterial community due to the strain H20-5 treatment, bulk and rhizosphere soil samples at 14 days after H20-5 treatment were used for Illumina MiSeq sequencing using the amplicon library of the V5-7 region of the 16S rRNA gene. From a total of 36 samples ( 3 salinity levels $\times 2$ treatments $\times 2$ compartments $\times 3$ replicates), 2,011,070 high-quality sequences were generated and clustered into 2,688 OTUs. For alpha-diversity analysis, the number of sequences was rarefied to 14,000 reads, which is the minimum number of reads among samples. The sequence depth was saturated at more than $97 \%$ coverage.

In the bulk soils, bacterial richness and diversity, represented by the Chaol richness estimate and Shannon's diversity index, were significantly reduced as soil EC levels increased, regardless of H20-5 treatment (Fig. 1A and C). In the rhizosphere soils, Chaol values were not signifi- 

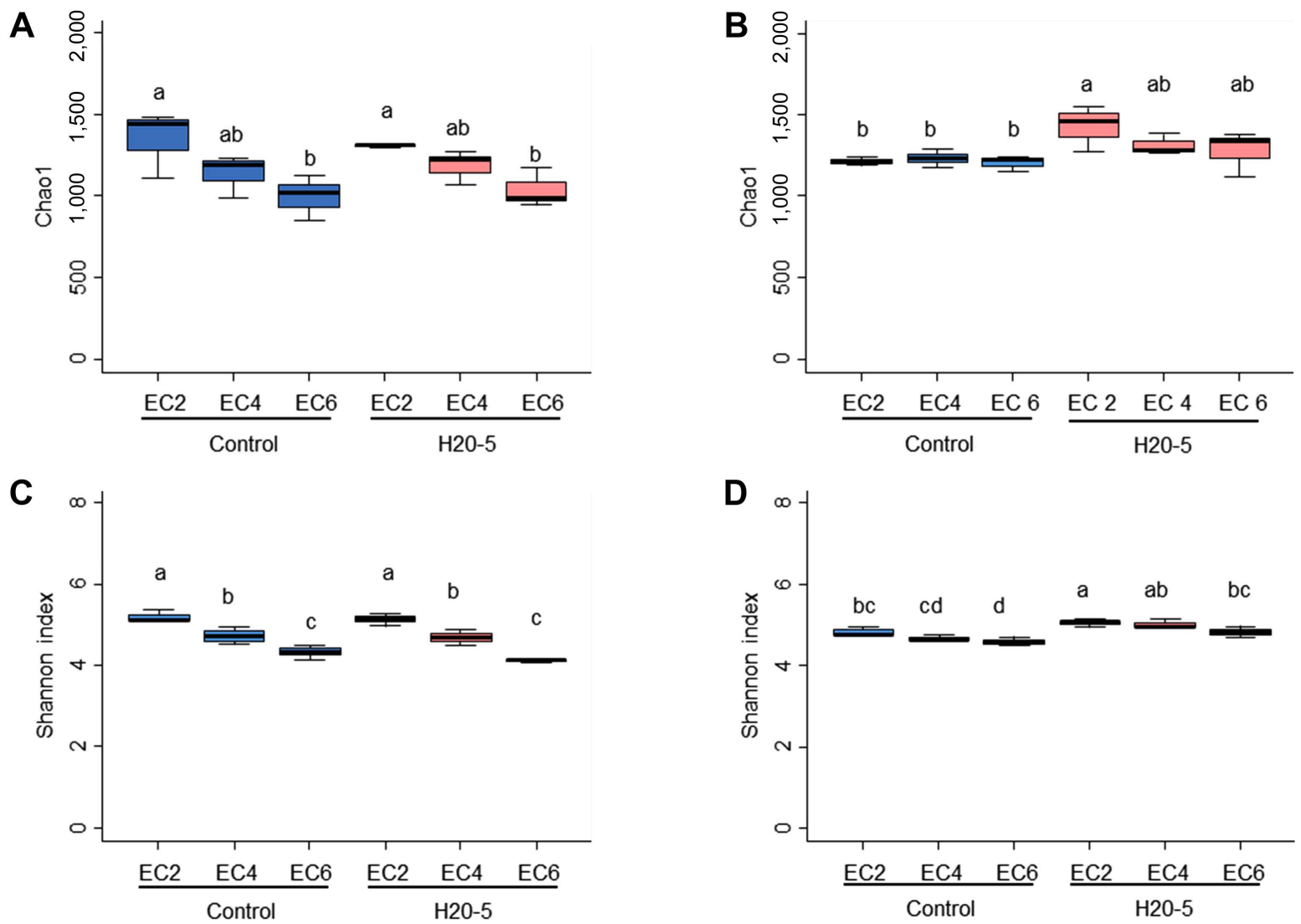

Fig. 1. Box plots showing the bacterial richness and diversity in bulk (A, C) and rhizosphere (B, D) soils 14 days after the Bacillus mesonae H20-5 treatment. Different letters indicate a significance by the least significant difference test at $P<0.05$.

cantly affected when soil EC levels were increased, and the EC value of $2 \mathrm{ds} / \mathrm{m}$ of soil treated with H20-5 was higher than that in the control (Fig. 1B). Although the patterns of the Shannon index changes were slightly decreased by increasing soil EC levels, overall values of Shannon index were higher in the H20-5 treated rhizosphere soil than in the control (Fig. 1D).

Effect of strain H20-5 on bacterial community assemblages. The bacterial community assemblages represented by NMDS biplots based on the Bray-Curtis dissimilarity were differentiated by rhizo-compartments (bulk and rhi-
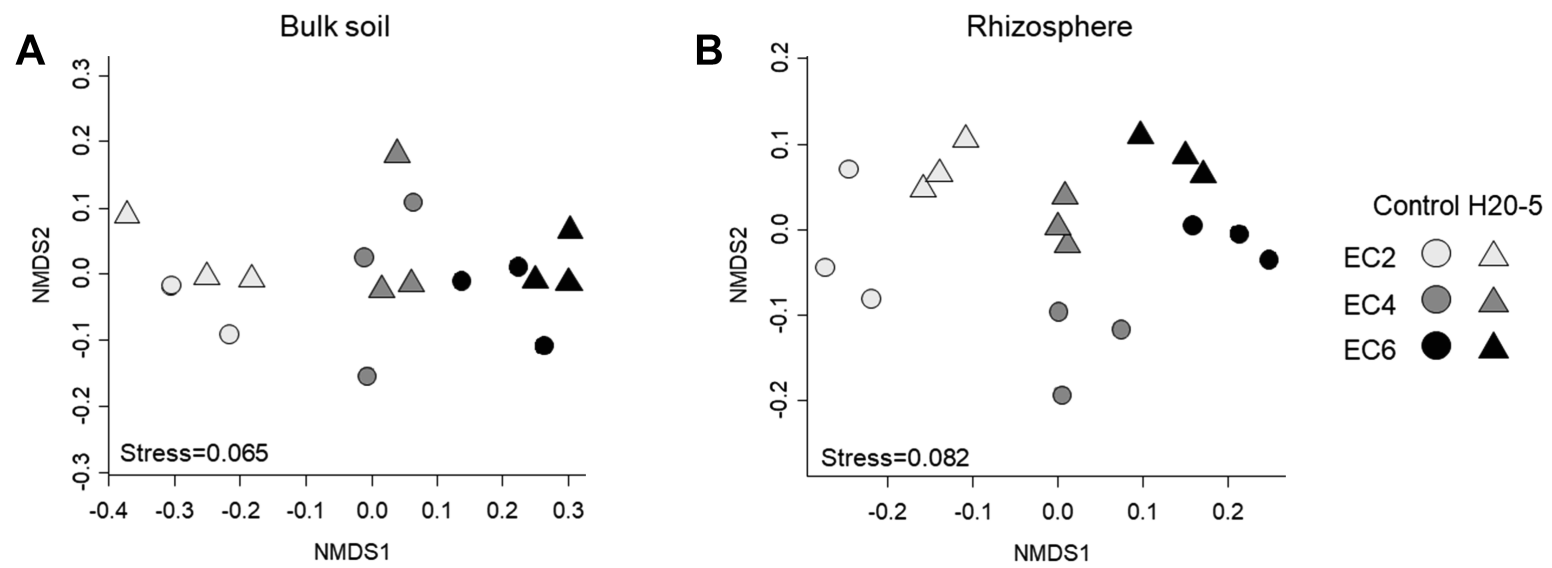

Fig. 2. Non-metric multidimensional scaling (NMDS) ordinations of bacterial community composition in the bulk (A) and rhizosphere (B) soils with and without Bacillus mesonae $\mathrm{H} 20-5$ treatment under different soil salinity levels. 
zosphere soils) along the y-axis (Supplementary Fig. 4). The bacterial community structures were clearly separated by EC levels along the X-axis in both the bulk and rhizosphere soils. The ordination results were further supported by a non-parametric analysis of similarities (ANOSIM) and PERMANOVA based on the Bray-Curtis dissimilarity (Supplementary Table 1). ANOSIM $(R=0.6278, P<0.001)$ and PERMANOVA $\left(R^{2}=0.3073, P<0.001\right)$ revealed a significant separation of the bacterial communities by soil EC levels. The ANOSIM $(R=0.4294, P<0.001)$ and PERMANOVA $\left(R^{2}=0.1523, P<0.001\right)$ results also demonstrated that the bacterial communities were significantly different in each rhizo-compartment.

To compare the shift in bacterial community upon $\mathrm{H} 20-5$ treatment in the bulk and rhizosphere soils, NMDS biplots were analyzed separately by rhizo-compartments (Fig. 2). Bacterial communities in the bulk and rhizosphere soils were clustered according to different soil EC levels. Intriguingly, the composition of bacterial communities in the H20-5 treated rhizosphere was similar regardless of the EC values, compared to the control. This suggests that strain H20-5 affects bacterial communities and is more stable toward the changes in salinity levels.

Effect of strain H20-5 on bacterial taxonomic distribution. Because the bacterial communities in the rhizosphere soils were more affected by H20-5 than those in the bulk soils, we focused on the composition of bacterial commu- nities in the rhizosphere samples. The most dominant phylum in the rhizosphere soils was Proteobacteria, followed by Actinobacteria, Acidobacteria, and Bacteroidetes (Fig. 3). The relative abundances of Alphaproteobacteria, Acidobacteria, and Gemmatimonadetes decreased with increasing soil EC levels in the rhizosphere soils of the control, whereas those of Gammaproteobacteria and Firmicutes gradually increased. The decreasing patterns of Alphaproteobacteria, Acidobacteria, and Gemmatimonadetes were diminished in the H20-5 treated rhizosphere soils.

A species indicator analysis was performed to identify OTUs enriched upon H20-5 treatments. Dominant OTUs $(>0.1 \%$ average relative abundance) with high indicator values $(>0.5)$ are listed in Supplementary Table 2. Only three OTUs exhibited higher relative abundances in the non-treated rhizosphere soils than in the H20-5 treatments. The relative abundances of 19 OTUs were higher in the H20-5 treated rhizosphere soils than in the control. Among the indicator OTUs, the relative abundance of OTU62 was increased upon H20-5 treatment, independent of the soil EC levels (Fig. 4). The relative abundances of OTU164, OTU131, OTU79, OTU119, and OTU436 were similar at an EC of $2 \mathrm{ds} / \mathrm{m}$ and gradually increased with soil EC levels in the rhizosphere upon $\mathrm{H} 20-5$ treatment. The relative abundance patterns of OTU81, OTU70, OTU90, OTU66, and OTU12 in the H20-5 treated rhizosphere soils were relatively stable over the different soil EC levels, whereas those in non-treated rhizosphere soils decreased with

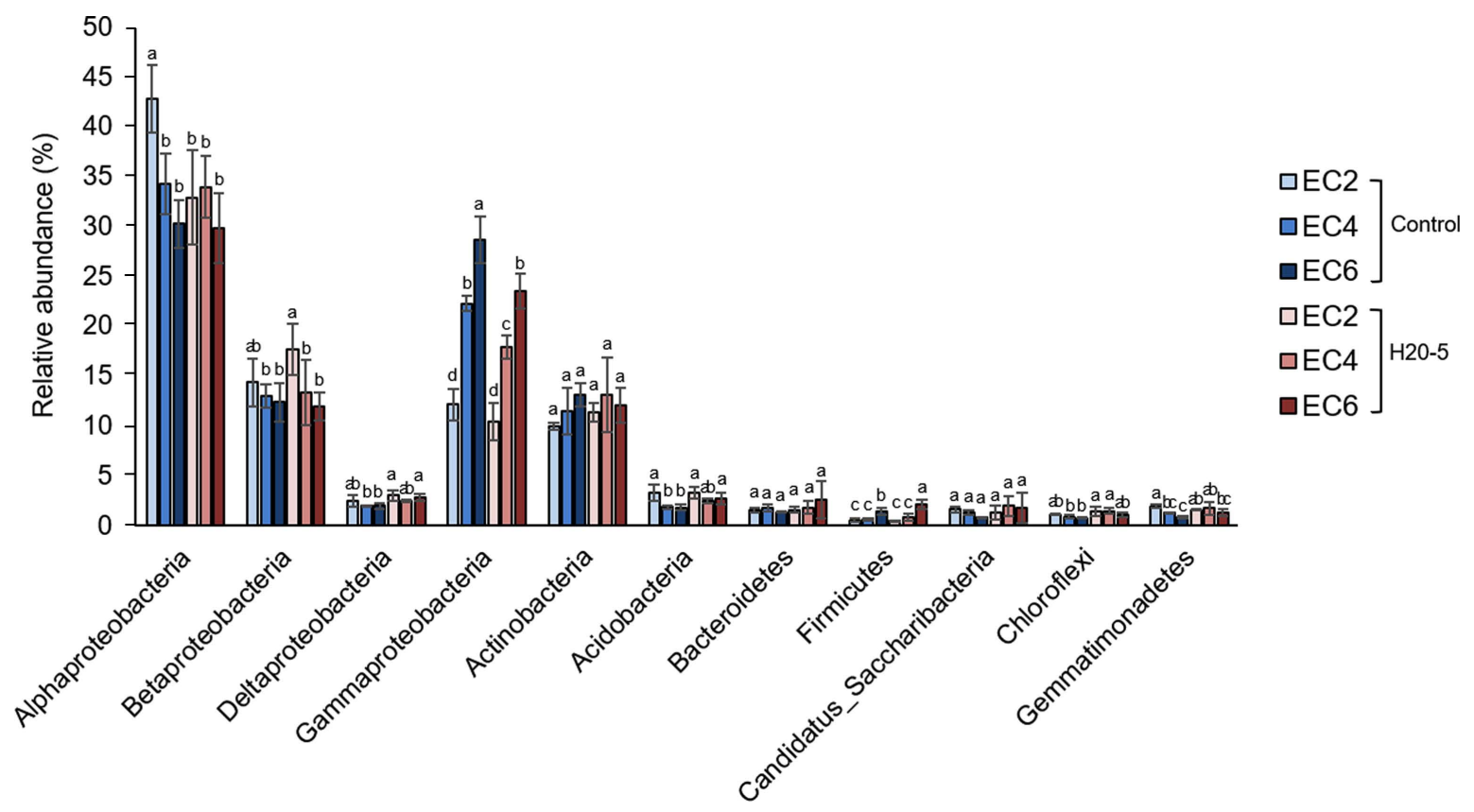

Fig. 3. Effect of Bacillus mesonae H20-5 on bacterial taxonomic distribution at phylum level in rhizospheres with different soil salinity levels. Error bars indicate standard error. Different letters indicate a significance by the least significant difference test at $P<0.05$. 

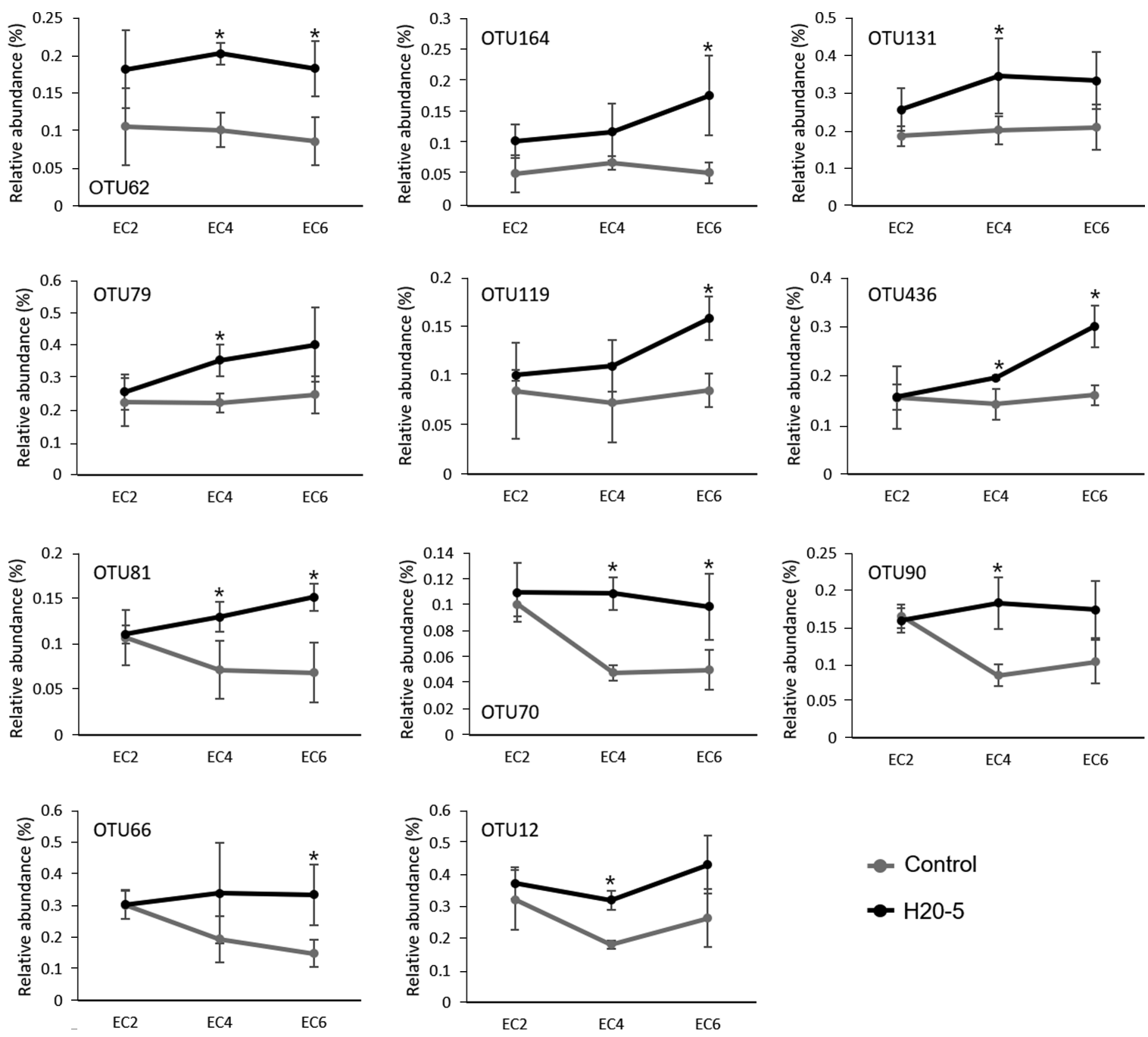

Control

$\mathrm{H} 20-5$

Fig. 4. Relative abundance of bacterial operational taxonomic units (OTUs) at different soil salinity levels. The asterisks indicate significant differences between the treatment and control determined by Student's t-test $(* P<0.05)$. Error bars indicate standard error.

increasing soil EC levels. Among these OTUs enriched by $\mathrm{H} 20-5$ treatment, eight OTUs were affiliated with $A c$ tinobacteria and four were Alphaproteobacteria. In the phylum Actinobacteria, several putative genera including Kineosporia, Virgisporangium, Actinoplanes, Gaiella, Blastococcus, and Solirubrobacter were enriched by H205 treatment. In the phylum Alphaproteobacteria, the genera Pseudolabrys, Bradyrhizobium, and Hyphomicrobium were enriched.

Effect of strain H20-5 on bacterial network. To understand the effect of strain $\mathrm{H} 20-5$ on bacterial interactions in the rhizosphere with different soil EC levels, co-occurrence network analysis was performed using an RMT-based network analysis. To compare the network structure, the input data were categorized as H20-5 treatment and control, and core OTUs that were present in all nine samples (three EC levels $\times$ three replicates) were used for network construction (Supplementary Fig. 5). The total number of the core OTUs was 702 and 431 in the H20-5 treated rhizosphere and control, respectively. The network connectivity in all compartments was well fitted by the power law, with an $R^{2}$ value $>0.7$, indicating scale-free properties. The numbers of nodes and modules, which were associated with the microbial co-occurrence network, were higher in the H20-5 treated rhizosphere soils. Although the average degree (avgK), which is the average number of connectors per node, was lower than that of the control (Table 1), the values of topological descriptors representing structural complexity of the network were higher in the H20-5 treated rhizosphere soils than in the control (Supplementary Table 3 ). This finding suggests that microbial communities in the 
Table 1. Topological properties of the networks of bacterial communities in the rhizosphere soils with and without $B$. mesonae H20-5 treatment

\begin{tabular}{lcc}
\hline Network index & Control & H20-5 \\
\hline No. of core OTUs & 431 & 702 \\
Total nodes & 307 & 492 \\
Tetal links & 1346 & 1839 \\
R of power-law & 0.75 & 0.856 \\
Average degree (avgK) & 8.769 & 7.476 \\
Average path distance (GD) & 3.949 & 4.316 \\
Average clustering coefficient (avgCC) & 0.278 & 0.185 \\
Modularity & 0.402 & 0.487 \\
No. of modules & 32 & 44 \\
No. of module hubs & 4 & 6 \\
No. of connectors & 11 & 22 \\
\hline
\end{tabular}

OUT, operational taxonomic units.

H20-5 treated rhizosphere soils formed complex networks than those in the control soil.

The treatment with strain H20-5 also affected the topological roles of the taxa in the network. The topological roles of the taxa in the network were classified into following four categories based on the values of within-module connectivity $(\mathrm{Zi})$ and among-module connectivity $(\mathrm{Pi})$ : peripherals, few interactions with other nodes $(\mathrm{Zi}<2.5$ and $\mathrm{Pi}<0.62)$; connectors, many links with other modules $(\mathrm{Zi}<2.5$ and $\mathrm{Pi}>0.62)$; module hubs, many interactions within the module $(\mathrm{Zi}>2.5$ and $\mathrm{Pi}<0.62)$; and network hubs, many interactions within and among modules $(\mathrm{Zi}>$ 2.5 and $\mathrm{Pi}>0.62$ ) (Supplementary Fig. 6). We identified six module hubs and 22 connectors in the bacterial network of H20-5 treated rhizosphere soils; however, the bacterial network of the control included four module hubs and 11 connectors (Supplementary Fig. 6). The higher numbers of module hubs and connectors in the bacterial network of H20-5 treatments suggested that more members in the soils were associated with the bacterial interactions induced by H20-5 treatment, although the average connectivity was reduced.

\section{Discussion}

Previous studies have revealed that Bacillus mesonae strain $\mathrm{H} 20-5$ promotes tomato growth under different salinity conditions, with soil EC levels of 2, 4, and $6 \mathrm{ds} / \mathrm{m}$. Although the copy numbers of H20-5 measured via qRTPCR were rapidly decreased in the bulk and rhizosphere soils, a shift in the bacterial community was observed upon H20-5 treatment.
Bacterial richness and diversity, represented by the Chaol and Shannon index values, were significantly reduced with increasing EC levels in the bulk and rhizosphere soils; however, they were not decreased in the H205 treated rhizosphere soils when compared to the control. The number of the core OTUs, which were detected in all the rhizosphere soil samples at different salinity levels, was also higher in the H20-5 treated rhizosphere soils. This is consistent with previous reports that bacterial species diversity in the rhizosphere and root endosphere was increased upon treatment with PGPRs belonging to the genus Bacillus (Gadhave et al., 2018; Zhang et al., 2019b). Considering that higher bacterial diversity was positively correlated with plant growth promotion (Garbeva et al., 2004; Liu et al., 2018), the bacterial community harboring more diverse bacterial members in the H20-5 treated rhizosphere enhanced plant growth in tomato.

NMDS analyses revealed that soil EC, which represents salinity, and rhizo-compartments, differentiated as bulk and rhizosphere soils, were the major factors shaping the microbial community structure. Soil EC is a critical factor not only for plant growth, but also for microbial community assembly (Casamayor et al., 2002; Kim et al., 2016; Min et al., 2016). Microbial community analysis with diverse soils across continents that have variations in edaphic factors, such as $\mathrm{pH}, \mathrm{EC}$, organic matter, and nitrogen, revealed that microbial community structure was highly correlated with pH and EC (Lee et al., 2019). Because the soil pH was unchanged in this study, a gradual shift was observed in the bacterial community according to soil EC levels. The diversity and composition of the microbial community in the rhizosphere were distinguished from those in bulk soil. The rhizosphere is a nutrient-rich region due to root exudation and rhizodeposition. The microorganisms that attract these compounds reshape their communities in the rhizosphere (Bulgarelli et al., 2013). A microbial community study of field soil cultivated with tomato plants also revealed that the microbial community structures were clearly differentiated by rhizo-compartments, regardless of soil type (Lee et al., 2019).

We observed that the effect of $\mathrm{H} 20-5$ treatment on the diversity and structure of the bacterial community was greater in the rhizosphere soil than in the bulk soil. Considering that the density of H20-5 in the bulk and rhizosphere soils was extremely low, strain H20-5 might have indirectly affected the bacterial communities through the tomato plants. The priming activity of the tomato plants exposed to H20-5 conferred tolerance to salinity stress, promoting the growth, quantity, and quality of the fruits (Yoo et al., 2019a, 2019b). The tomato plants IST upon H20-5 treat- 
ment, which changed its physiology. The tomato plants treated with $\mathrm{H} 20-5$ exhibited increased levels of $\mathrm{Ca}^{2+}$, proline, and $\mathrm{ABA}$ and enhanced antioxidant enzyme activities, including catalase, superoxide dismutase, and glutathione peroxidase activities compared to the controls (Yoo et al., 2019b). These physiological changes could lead to differences in the composition of root exudates between tomato plants with and without H20-5 treatment, which affects the bacterial community. There were variations in the bacterial communities associated with different Arabidopsis mutants related to biosynthesis and signaling pathways of plant hormones, including ABA and jasmonic acid (Carvalhais et al., 2013; Lebeis et al., 2015; Liu et al., 2017). The microbial communities in the rhizosphere differ according to crop species and plant development, owing to the varying compositions of root exudates (Chaparro et al., 2014; Edwards et al., 2015, 2018). Greater diversity and richness of bacterial communities in the H20-5 treated rhizosphere soil indicated that the H20-5 treated tomato plants recruited more bacterial members around the soil.

The relative abundances of Alphaproteobacteria, Acidobacteria, and Gemmatimonadetes were gradually decreased depending on soil EC levels in the non-treated rhizosphere soils, and they were relatively stable in the H20-5 treated rhizosphere soils. NMDS analysis revealed that the variation in the structures of bacterial communities with increasing soil EC was relatively less in the H20-5 treated rhizosphere soils compared to the control. These results suggest that the H20-5 treated bacterial communities were relatively stable compared to the control. Microbial communities rapidly respond to environmental changes; however, the bacterial communities in the H20-5 treated rhizosphere soils minimized the environmental effect by conferring tolerance to salinity stress on tomato plants.

We identified enriched OTUs belonging to Steroidobacter, Kineosporia, Virgisporangium, Nitrospira, Pseudolabrys, Gaiella, Bradyrhizobium, Hyphomicrobium, Blastococcus, and Solirubrobacte upon H20-5 treatment under salinity stress conditions. The relative abundance of Steroidobacter, Gaiella, and Pseudolabrys was significantly altered by increasing salinity in the rhizosphere soil of Jerusalem artichoke (Yang et al., 2016). Bradyrhizobium is well-known for nodulation and nitrogen fixation in leguminous plants, and it promotes the growth of non-leguminous plants (Antoun et al., 1998). Bacillus spp. improved plant growth by recruiting other bacterial members from the soil. For example, Bacillus amyloliquefaciens enhanced soybean growth and nodulation by recruiting the natural symbiont Bradyrhizobium japonicum (Masciarelli et al., 2014).

Bacterial co-occurrence networks in the rhizosphere with different soil EC levels indicated that the structural complexity of the network quantified by topological descriptors was higher in the H20-5 treatment group than in the control despite the lower average degree. A relatively large number of nodes with low degree in the H20-5 treatment group networks would result in an overall low average degree. Despite this low value, more bacterial members were associated in the bacterial network of the H20-5 treated rhizosphere soils than in the control. The number of modules, which are the groups of nodes that are highly connected to each other, and keystone taxa representing module hubs and connectors, which play potential roles in the network structure, were relatively higher in the $\mathrm{H} 20-5$ treated rhizosphere soils than in the control. H20-5 likely has an impact on the dispersion of ecological functional processes into various modules. Efficient communication among different members of microbial communities can effect rapid responses to environmental changes (Deng et al., 2012). Modularity is an important concept in ecology for system stability and resilience (Olesen et al., 2007). Overall, the bacterial community affected by strain H20-5 might be relatively stable to salinity changes.

In summary, B. mesonae H20-5 IST of tomato plants under salinity stress, which affected the structure and composition of the soil bacterial community. The bacterial species richness and diversity in the rhizosphere were increased upon H20-5 treatment. There were no abrupt changes in the composition of bacterial communities at different salinity levels in the H20-5 treated rhizosphere soils compared to control. More bacterial species and more modules of the bacterial network were identified in the H20-5 treated rhizosphere soil compared to the control. These findings demonstrate the effects of introducing PGPB on bacterial communities under different salinity conditions. The results suggest that not only plant physiology, but also the diversity and structure of the microbial community, should be considered to improve sustainable crop productivity under changing environmental conditions.

\section{Conflicts of Interest}

No potential conflict of interest relevant to this article was reported.

\section{Acknowledgments}

This research was carried out with the support of "Cooperative Research Program for Agricultural Science \& Technology Development (Project No. PJ01424401)", Rural Development Administration, South Korea. 


\section{Electronic Supplementary Material}

Supplementary materials are available at The Plant Pathology Journal website (http://www.ppjonline.org/).

\section{References}

Anderson, M. J. 2001. A new method for non-parametric multivariate analysis of variance. Aust. Ecol. 26:32-46.

Antoun, H., Beauchamp, C. J., Goussard, N., Chabot, R. and Lalande, R. 1998. Potential of Rhizobium and Bradyrhizobium species as plant growth promoting rhizobacteria on nonlegumes: effect on radishes (Raphanus sativus L.). Plant Soil 204:57-67.

Backer, R., Rokem, J. S., Ilangumaran, G., Lamont, J., Praslickova, D., Ricci, E., Subramanian, S. and Smith, D. L. 2018. Plant growth-promoting rhizobacteria: context, mechanisms of action, and roadmap to commercialization of biostimulants for sustainable agriculture. Front. Plant Sci. 9:1473.

Bulgarelli, D., Schlaeppi, K., Spaepen, S., Ver Loren van Themaat, E. and Schulze-Lefert, P. 2013. Structure and functions of the bacterial microbiota of plants. Annu. Rev. Plant Biol. 64:807-838.

Carvalhais, L. C., Dennis, P. G., Badri, D. V., Tyson, G. W., Vivanco, J. M. and Schenk, P. M. 2013. Activation of the jasmonic acid plant defence pathway alters the composition of rhizosphere bacterial communities. PLoS ONE 8:e56457.

Casamayor, E. O., Massana, R., Benlloch, S., Øvreås, L., Díez, B., Goddard, V. J., Gasol, J. M., Joint, I., Rodríguez-Valera, F. and Pedrós-Alió, C. 2002. Changes in archaeal, bacterial and eukaryal assemblages along a salinity gradient by comparison of genetic fingerprinting methods in a multipond solar saltern. Environ. Microbiol. 4:338-348.

Chaparro, J. M., Badri, D. V. and Vivanco, J. M. 2014. Rhizosphere microbiome assemblage is affected by plant development. ISME J. 8:790-803.

Clarke, K. R. 1993. Nonparametric multivariate analyses of changes in community structure. Aust. J. Ecol. 18:117-143.

Cole, J. R., Wang, Q., Fish, J. A., Chai, B., McGarrell, D. M., Sun, Y., Brown, C. T., Porras-Alfaro, A., Kuske, C. R. and Tiedje, J. M. 2014. Ribosomal Database Project: data and tools for high throughput rRNA analysis. Nucleic Acids Res. 42:D633-D642.

Compant, S., Samad, A., Faist, H. and Sessitsch, A. 2019. A review on the plant microbiome: ecology, functions, and emerging trends in microbial application. J. Adv. Res. 19:2937.

De Cáceres, M. and Legendre, P. 2009. Associations between species and groups of sites: indices and statistical inference. Ecology 90:3566-3574.

Deng, Y., Jiang, Y.-H., Yang, Y., He, Z., Luo, F. and Zhou, J. 2012. Molecular ecological network analyses. BMC Bioinform. 13:113.

Edgar, R. C. 2013. UPARSE: highly accurate OTU sequences from microbial amplicon reads. Nat. Methods 10:996-998.

Edwards, J., Johnson, C., Santos-Medellin, C., Lurie, E., Podishetty, N. K., Bhatnagar, S., Eisen, J. A. and Sundaresan, V. 2015. Structure, variation, and assembly of the rootassociated microbiomes of rice. Proc. Natl. Acad. Sci. U. S. A. 112:E911-E920.

Edwards, J. A., Santos-Medellín, C. M., Liechty, Z. S., Nguyen, B., Lurie, E., Eason, S., Phillips, G. and Sundaresan, V. 2018. Compositional shifts in root-associated bacterial and archaeal microbiota track the plant life cycle in field-grown rice. PLoS Biol. 16:e2003862.

Gadhave, K. R., Devlin, P. F., Ebertz, A., Ross, A. and Gange, A. C. 2018. Soil inoculation with Bacillus spp. modifies root endophytic bacterial diversity, evenness, and community composition in a context-specific manner. Microb. Ecol. 76:741750.

Garbeva, P., van Veen, J. A. and van Elsas, J. D. 2004. Microbial diversity in soil: selection microbial populations by plant and soil type and implications for disease suppressiveness. Annu. Rev. Phytopathol. 42:243-270.

Glick, B. R. 2012. Plant growth-promoting bacteria: mechanisms and applications. Scientifica (Cairo) 2012:963401.

Jamil, A., Riaz, S., Ashraf, M. and Foolad, M. R. 2011. Gene expression profiling of plants under salt stress. Crit. Rev. Plant Sci. 30:435-458.

Kim, J. M., Roh, A.-S., Choi, S.-C., Kim, E.-J., Choi, M.-T., Ahn, B.-K., Kim, S.-K., Lee, Y.-H., Joa, J.-H., Kang, S.-S., Lee, S. A., Ahn, J.-H., Song, J. and Weon, H.-Y. 2016. Soil pH and electrical conductivity are key edaphic factors shaping bacterial communities of greenhouse soils in Korea. J. Microbiol. 54:838-845.

Lebeis, S. L., Paredes, S. H., Lundberg, D. S., Breakfield, N., Gehring, J., McDonald, M., Malfatti, S., Glavina del Rio, T., Jones, C. D., Tringe, S. G. and Dangl, J. L. 2015. PLANT MICROBIOME: salicylic acid modulates colonization of the root microbiome by specific bacterial taxa. Science 349:860864.

Lee, S. A., Kim, Y., Kim, J. M., Chu, B., Joa, J.-H., Sang, M. K., Song, J. and Weon, H.-Y. 2019. A preliminary examination of bacterial, archaeal, and fungal communities inhabiting different rhizocompartments of tomato plants under real-world environments. Sci. Rep. 9:9300.

Legendre, P. and Gallagher, E. D. 2001. Ecologically meaningful transformations for ordination of species data. Oecologia 129:271-280.

Liu, H., Carvalhais, L. C., Schenk, P. M. and Dennis, P. G. 2017. Effects of jasmonic acid signalling on the wheat microbiome differ between body sites. Sci. Rep. 7:41766.

Liu, H., Xiong, W., Zhang, R., Hang, X., Wang, D., Li, R. and Shen, Q. 2018. Continuous application of different organic additives can suppress tomato disease by inducing the healthy rhizospheric microbiota through alterations to the bulk soil microflora. Plant Soil 423:229-240.

Masciarelli, O., Llanes, A. and Luna, V. 2014. A new PGPR co- 
inoculated with Bradyrhizobium japonicum enhances soybean nodulation. Microbiol. Res. 169:609-615.

Min, W., Guo, H., Zhang, W., Zhou, G., Ma, L., Ye, J., Liang, Y. and Hou, Z. 2016. Response of soil microbial community and diversity to increasing water salinity and nitrogen fertilization rate in an arid soil. Acta Agric. Scand. Sect. B Soil Plant Sci. 66:117-126.

Mueller, L. A., Kugler, K. G., Dander, A., Graber, A. and Dehmer, M. 2011. QuACN: an R package for analyzing complex biological networks quantitatively. Bioinformatics 27:140-141.

Munns, R. 2005. Genes and salt tolerance: bringing them together. New Phytol. 167:645-663.

Oksanen, J., Blanchet, F. G., Kindt, R., Legendre, P., Minchin, P. R., O'Hara, R. B., Simpson, G. L., Solymos, P., Stevens, M. H. H. and Wagner, H. 2013. Vegan: community ecology package. R package version 2.0-10. R Foundation for Statistical Computing, Vienna, Austria.

Olesen, J. M., Bascompte, J., Dupont, Y. L. and Jordano, P. 2007. The modularity of pollination networks. Proc. Natl. Acad. Sci. U. S. A. 104:19891-19896.

Panke-Buisse, K., Poole, A. C., Goodrich, J. K., Ley, R. E. and Kao-Kniffin, J. 2015. Selection on soil microbiomes reveals reproducible impacts on plant function. ISME J. 9:980-989.

Polonenko, D. R., Mayfield, C. I. and Dumbroff, E. B. 1986. Microbial responses to salt-induced osmotic stress. Plant Soil 92:417-425.

R Development Core Team. 2014. R: a language and environment for statistical computing. URL http://www.R-project. org/ [21 October 2021].

Sawant, S. S., Kim, S. Y., Sang, M. K., Weon, H.-Y., Kim, S. and Song, J. 2019. Complete genome sequence of Bacillus mesonae $\mathrm{H} 20-5$, an efficient strain enhancing abiotic stress tolerance in plants. Korean J. Microbiol. 55:408-410.

Schloss, P. D., Westcott, S. L., Ryabin, T., Hall, J. R., Hartmann, M., Hollister, E. B., Lesniewski, R. A., Oakley, B. B., Parks, D. H., Robinson, C. J., Sahl, J. W., Stres, B., Thallinger, G. G., Van Horn, D. J. and Weber, C. F. 2009. Introducing mothur: open-source, platform-independent, community-supported software for describing and comparing microbial communities. Appl. Environ. Microbiol. 75:7537-7541.

Shi, S., Nuccio, E. E., Shi, Z. J., He, Z., Zhou, J. and Firestone, M. K. 2016. The interconnected rhizosphere: high network complexity dominates rhizosphere assemblages. Ecol. Lett. 19:926-936.

Shrivastava, P. and Kumar, R. 2015. Soil salinity: a serious environmental issue and plant growth promoting bacteria as one of the tools for its alleviation. Saudi J. Biol. Sci. 22:123-131.

Tao, C., Li, R., Xiong, W., Shen, Z., Liu, S., Wang, B., Ruan, Y., Geisen, S., Shen, Q. and Kowalchuk, G. A. 2020. Bio-organic fertilizers stimulate indigenous soil Pseudomonas populations to enhance plant disease suppression. Microbiome 8:137.
Toju, H., Peay, K. G., Yamamichi, M., Narisawa, K., Hiruma, K., Naito, K., Fukuda, S., Ushio, M., Nakaoka, S., Onoda, Y., Yoshida, K., Schlaeppi, K., Bai, Y., Sugiura, R., Ichihashi, Y., Minamisawa, K. and Kiers, E. T. 2018. Core microbiomes for sustainable agroecosystems. Nat. Plants 4:247-257.

Trabelsi, D. and Mhamdi, R. 2013. Microbial inoculants and their impact on soil microbial communities: a review. Biomed. Res. Int. 2013:863240.

Wang, L., Lu, X., Yuan, H., Wang, B. and Shen, Q. 2015. Application of bio-organic fertilizer to control tomato fusarium wilting by manipulating soil microbial communities and development. Commun. Soil Sci. Plant Anal. 46:2311-2322.

Wang, Q., Garrity, G. M., Tiedje, J. M. and Cole, J. R. 2007. Naive Bayesian classifier for rapid assignment of rRNA sequences into the new bacterial taxonomy. Appl. Environ. Microbiol. 73:5261-5267.

Wei, Z., Gu, Y., Friman, V.-P., Kowalchuk, G. A., Xu, Y., Shen, Q. and Jousset, A. 2019. Initial soil microbiome composition and functioning predetermine future plant health. Sci. $A d v$. 5:eaaw0759.

Xue, C., Penton, C. R., Shen, Z., Zhang, R., Huang, Q., Li, R., Ruan, Y. and Shen, Q. 2015. Manipulating the banana rhizosphere microbiome for biological control of Panama disease. Sci. Rep. 5:11124.

Yang, H., Hu, J., Long, X., Liu, Z. and Rengel, Z. 2016. Salinity altered root distribution and increased diversity of bacterial communities in the rhizosphere soil of Jerusalem artichoke. Sci. Rep. 6:20687.

Yang, J., Kloepper, J. W. and Ryu, C.-M. 2009. Rhizosphere bacteria help plants tolerate abiotic stress. Trends Plant Sci. 14:14.

Yoo, S.-J., Kim, J. W., Kim, S. T., Weon, H.-Y., Song, J. and Sang, M. K. 2019a. Effect of Bacillus mesonae H20-5 on fruit yields and quality in protected cultivation. Res. Plant Dis. 25:84-88.

Yoo, S.-J., Weon, H.-Y., Song, J. and Sang, M. K. 2019b. Induced tolerance to salinity stress by halotolerant bacteria Bacillus aryabhattai $\mathrm{H} 19-1$ and B. mesonae $\mathrm{H} 20-5$ in tomato plants. $J$. Microbiol. Biotechnol. 29:1124-1136.

Zhang, L.-N., Wang, D.-C., Hu, Q., Dai, X.-Q., Xie, Y.-S., Li, Q., Liu, H.-M. and Guo, J.-H. 2019a. Consortium of plant growth-promoting rhizobacteria strains suppresses sweet pepper disease by altering the rhizosphere microbiota. Front. Microbiol. 10:1668.

Zhang, Y., Gao, X., Shen, Z., Zhu, C., Jiao, Z., Li, R. and Shen, Q. 2019b. Pre-colonization of PGPR triggers rhizosphere microbiota succession associated with crop yield enhancement. Plant Soil 439:553-567.

Zolla, G., Badri, D. V., Bakker, M. G., Manter, D. K. and Vivanco, J. M. 2013. Soil microbiomes vary in their ability to confer drought tolerance to Arabidopsis. Appl. Soil Ecol. 68:1-9. 